\title{
Some Results on Fuzzy Soft Topological Spaces
}

\author{
Cigdem Gunduz (Aras) ${ }^{1,2}$ and Sadi Bayramov ${ }^{1,2}$ \\ ${ }^{1}$ Department of Mathematics, Kocaeli University, Kocaeli 41380, Turkey \\ ${ }^{2}$ Department of Mathematics, Kafkas University, Kars 36100, Turkey
}

Correspondence should be addressed to Sadi Bayramov; baysadi@gmail.com

Received 24 February 2013; Accepted 22 April 2013

Academic Editor: Safa Bozkurt Coskun

Copyright (C) 2013 C. Gunduz (Aras) and S. Bayramov. This is an open access article distributed under the Creative Commons Attribution License, which permits unrestricted use, distribution, and reproduction in any medium, provided the original work is properly cited.

\begin{abstract}
We introduce some important properties of fuzzy soft topological spaces. Furthermore, fuzzy soft continuous mapping, fuzzy soft open and fuzzy soft closed mappings, and fuzzy soft homeomorphism for fuzzy soft topological spaces are given and structural characteristics are discussed and studied.
\end{abstract}

\section{Introduction}

In many complicated problems arising in the fields of economics, social sciences, engineering, medical science, and so forth. involving uncertainties, classical methods are found to be inadequate in latest year. A number of theories have been proposed for dealing with uncertainties in an efficient way like theory of fuzzy sets [1], theory of intuitionistic fuzzy sets [2], theory of vague sets, theory of interval mathematics [3,4], and theory of rough sets [5]. However, these theories have their own difficulties.

In 1999, Molodtsov [6] introduced the concept of soft set which is completely new approach for modeling uncertainties. He applied several directions for the applications of soft sets, such as smoothness of functions, game theory, Riemann-integration, and theory of probability. Now, soft set theory and its applications are progressing rapidly in different fields. Maji et al. [7, 8] gave some new definitions on soft sets and presented an application of soft sets in decision making problems. Chen et al. [9], Feng et al. [10], Aktaş and Çağman [11], Sun et al. [12], and Ali et al. [13] improved the works of Maji et al. [7] that initiated the study involving both fuzzy sets and soft sets. Later some researchers studied the concept of fuzzy soft sets [14-17]. Moreover, Shabir and Naz [18] presented soft topological spaces and defined some concepts of soft sets on this spaces and separation axioms. Later Tanay and Kandemir [19] initially gave the concept of fuzzy soft topology using the fuzzy soft sets and gave the basic notions of it by following Chang [20]. Pazar Varol and Aygun [21] defined fuzzy soft topology in Lowen's sense.

The purpose of this paper is to discuss some important properties of fuzzy soft topological spaces. Firstly, we recall some basic definitions in fuzzy soft sets and the results from the literature. The newly introduced concept of parameters comes into play with the collection of parameterization fuzzy topologies on the initial universe. Later we investigate the properties of fuzzy soft interior and fuzzy soft closure of fuzzy sets. We can say that a fuzzy soft topological space gives a parameterized family of fuzzy topologies on the initial universe, but the converse is not true. Finally fuzzy soft continuous mapping, fuzzy soft open and fuzzy soft closed mappings, and fuzzy soft homeomorphism for fuzzy soft topological spaces are investigated, and some interesting results that may be of value for further research are obtained.

\section{Preliminaries}

In this section, we will introduce necessary definitions and theorems for fuzzy soft sets.

Definition 1 (see [6]). Let $X$ be an initial universe set and $E$ be a set of parameters. A pair $(F, E)$ is called a soft set over $X$ if and only if $F$ is a mapping from $E$ into the set of all subsets of the set $X$, that is, $F: E \rightarrow P(X)$, where $P(X)$ is the power set of $X$. 
Definition 2 (see [15]). Let $I^{X}$ denote the set of all fuzzy sets on $X$ and $A \subset E$. A pair $(f, A)$ is called a fuzzy soft set over $X$, where $f$ is a mapping from $A$ into $I^{X}$. That is, for each $a \in A, f(a)=f_{a}: X \rightarrow I$ is a fuzzy set on $X$.

Definition 3 (see [15]). For two fuzzy soft sets $(f, A)$ and $(g, B)$ over a common universe $X$, we say that $(f, A)$ is a fuzzy soft subset of $(g, B)$ if

(i) $A \subset B$,

(ii) for each $a \in A, f_{a} \leq g_{a}$; that is, $f_{a}$ is a fuzzy subset of $g_{a}$.

This relationship is denoted by $(f, A) \tilde{\subset}(g, B)$. Similarly, $(f, A)$ is said to be a fuzzy soft superset of $(g, B)$ if $(g, B)$ is a fuzzy soft subset of $(f, A)$. This relationship is denoted by $(f, A) \widetilde{\jmath}(g, B)$.

Definition 4 (see [15]). Two fuzzy soft sets $(f, A)$ and $(g, B)$ over a common universe $X$ are said to be fuzzy soft equal if $(f, A)$ is a fuzzy soft subset of $(g, B)$ and $(g, B)$ is a fuzzy soft subset of $(f, A)$.

Definition 5 (see [15]). The union of two fuzzy soft sets $(f, A)$ and $(g, B)$ over a common universe $X$ is the fuzzy soft set $(h, C)$, where $C=A \cup B$, and, for all $c \in C$,

$$
h_{c}= \begin{cases}f_{c}, & \text { if } c \in A-B \\ g_{c}, & \text { if } c \in B-A \\ f_{c} \vee g_{c}, & \text { if } c \in A \cap B .\end{cases}
$$

This relationship is denoted by $(f, A) \widetilde{\cup}(g, B)=(h, C)$.

Definition 6 (see [15]). The intersection of two fuzzy soft sets $(f, A)$ and $(g, B)$ over a common universe $X$ is the fuzzy soft set $(h, C)$, where $C=A \cap B$, and, for all $c \in C, h_{c}=f_{c} \wedge g_{c}$. This is denoted by $(f, A) \widetilde{\cap}(g, B)=(h, C)$.

Definition 7 (see [14]). A fuzzy soft set $(f, A)$ over $U$ is said to be a null fuzzy soft set and is denoted by $\widetilde{\Phi}$ if and only if, for each $e \in A, f_{e}=\widetilde{0}$, where $\widetilde{0}$ is the membership function of null fuzzy set over $U$, which takes value 0 for all $x$ in $U$.

Definition 8 (see [14]). A fuzzy soft set $(f, A)$ over $U$ is said to be an absolute fuzzy soft set and is denoted by $\widetilde{U}$ if and only if, for each $e \in A, f_{e}=\widetilde{1}$, where $\tilde{1}$ is the membership function of absolute fuzzy set over $U$, which takes value 1 for all $x$ in $U$.

Definition 9 (see [15]). The complement of a fuzzy soft set $(f, A)$ is the fuzzy soft set $\left(f^{\prime}, A\right)$, which is denoted by $(f, A)^{\prime}$ and where $f^{\prime}: A \rightarrow F(U)$ is a fuzzy set-valued function that is, for each $a \in A, f^{\prime}(a)$ is a fuzzy set in $U$, whose membership function $f_{a}^{\prime}(x)=1-f_{a}(x)$ for all $x \in U$. Here $f_{a}^{\prime}$ is the membership function of $f^{\prime}(a)$.

Let $\left\{\left(f^{k}, A_{k}\right) \mid k \in K, A_{k} \subset E\right\}$ be a family of fuzzy soft sets over $U$ where, for each $k \in K, f^{k}: A_{k} \rightarrow F(U)$ is a fuzzy set-valued function, and each member $\left(f^{k}, A_{k}\right)$ of this family is denoted $(f, A)_{k}$, that is, for each $k \in K,(f, A)_{k}=\left(f^{k}, A_{k}\right)$.
Then the union and the intersection of this family are defined as follows.

Definition 10 (see $[10,14]$ ). Let $\left\{A_{k} \mid k \in K\right\} \subset P(E)$. The union of nonempty family $\left\{(f, A)_{k} \mid k \in K\right\}$ of fuzzy soft sets over a common universe $U$, denoted by $\widetilde{U}_{k \in K}(f, A)_{k}$, is defined as the fuzzy soft set $(h, C)$ such that $C=\cup_{k \in K} A_{k}$ and for each $c \in C, h_{c}(x)=\vee_{k \in K_{c}} f_{c}^{k}(x)$, for all $x \in U$, where $K_{c}=\left\{k \in K \mid c \in A_{k}\right\}$. Here $f_{c}^{k}$ is the membership function of the fuzzy set $f^{k}(c)$ for each $c \in C$.

Definition 11 (see $[10,14]$ ). Let $\left\{A_{k} \mid k \in K\right\} \subset P(E)$. The intersection of nonempty family $\left\{(f, A)_{k} \mid k \in K\right\}$ of fuzzy soft sets over a common universe $U$, denoted by $\widetilde{\cap}_{k \in K}(f, A)_{k}$, is defined as the fuzzy soft set $(h, C)$ such that $C=\cap_{k \in K} A_{k}$ and for each $c \in C, h_{c}(x)=\wedge_{k \in K_{c}} f_{c}^{k}(x)$, for all $x \in U$, where $K_{c}=\left\{k \in K \mid c \in A_{k}\right\}$. Here $f_{c}^{k}$ is the membership function of the fuzzy set $f^{k}(c)$ for each $c \in C$.

Definition 12 (see [18]). Let $\tau$ be the collection of soft sets over $X$, then $\tau$ is said to be a soft topology on $X$ if

(1) $\Phi, \widetilde{X}$ belong to $\tau$,

(2) the union of any number of soft sets in $\tau$ belongs to $\tau$,

(3) the intersection of any two soft sets in $\tau$ belongs to $\tau$.

The triplet $(X, \tau, E)$ is called a soft topological space over $X$. Let $F(X, E)$ be the family of all fuzzy soft sets over $X$.

Definition 13 (see [19]). A fuzzy soft topological space is a pair $(X, \tau)$ where $X$ is a nonempty set and $\tau$ is a family of fuzzy soft sets over $X$ satisfying the following properties:

(i) $\widetilde{\Phi_{E}}, \widetilde{1_{E}} \in \tau$,

(ii) if $f_{A}, g_{B} \in \tau$, then $f_{A} \cap g_{B} \in \tau$,

(iii) if $\left(f_{A}\right)_{i} \in \tau$, for all $i \in J$, then $\bigcup_{i \in J}\left(f_{A}\right)_{i} \in \tau$.

$\tau$ is called a topology of fuzzy soft sets on $X$. Every member of $\tau$ is called fuzzy soft open set. A fuzzy soft set is called $\tau$ closed if and only if its complement is $\tau$-open.

Definition 14 (see [21]). Let $(X, \tau)$ be a fuzzy soft topological space and $f_{A} \in F(X, E)$. The fuzzy soft closure of $f_{A}$, denoted $\overline{f_{A}}$, is the intersection of all fuzzy soft closed supersets of $f_{A}$.

Definition 15 (see [21]). Let $(X, \tau)$ be a fuzzy soft topological space and $f_{A} \in F(X, E)$. The fuzzy soft interior of $f_{A}$ denoted by $f_{A}^{\circ}$, is the union of all fuzzy soft open subsets of $f_{A}$.

\section{Fuzzy Soft Topology}

Tanay and Kandemir [19] introduced the notion of fuzzy soft topology. Now we introduce some important properties of fuzzy soft topological spaces and define the fuzzy soft closure (fuzzy soft boundary) of a fuzzy soft set. From now on, let $X$ be an initial universe set and $E$ be the nonempty set of parameters $(F, E)$ be a fuzzy soft set. Let $\widetilde{P}(X)$ denote the set of all fuzzy sets on $X$. 
Proposition 16 (see [21]). Let $(X, \tau, E)$ be a fuzzy soft topological space over $X$. Then the collection $\tau_{\alpha}=\{F(\alpha) \mid(F, E) \in \tau\}$, for each $\alpha \in E$, defines a fuzzy topology on $X$.

Proposition 16 shows that, corresponding to each parameter $\alpha \in E$, we have a fuzzy topology $\tau_{\alpha}$ on $X$. Thus a fuzzy soft topology on $X$ gives a parameterized family of fuzzy topologies on $X$.

Example 17. Let $X=\left\{x_{1}, x_{2}, x_{3}\right\}, E=\left\{e_{1}, e_{2}\right\}$, and $\tau=$ $\left\{\Phi, 1,\left(F_{1}, E\right),\left(F_{2}, E\right),\left(F_{3}, E\right),\left(F_{4}, E\right)\right\}$, where $\left(F_{1}, E\right),\left(F_{2}, E\right)$, $\left(F_{3}, E\right)$, and $\left(F_{4}, E\right)$ are fuzzy soft sets over $X$, for $1 \leq i \leq 4$, be defined as follows:

$$
\begin{array}{lll}
F_{1}\left(e_{1}\right)\left(x_{1}\right)=\frac{1}{2}, & F_{1}\left(e_{1}\right)\left(x_{2}\right)=\frac{1}{3}, & F_{1}\left(e_{1}\right)\left(x_{3}\right)=\frac{1}{4}, \\
F_{1}\left(e_{2}\right)\left(x_{1}\right)=\frac{1}{3}, & F_{1}\left(e_{2}\right)\left(x_{2}\right)=\frac{1}{2}, & F_{1}\left(e_{2}\right)\left(x_{3}\right)=\frac{1}{4}, \\
F_{2}\left(e_{1}\right)\left(x_{1}\right)=1, & F_{2}\left(e_{1}\right)\left(x_{2}\right)=0, & F_{2}\left(e_{1}\right)\left(x_{3}\right)=\frac{1}{2}, \\
F_{2}\left(e_{2}\right)\left(x_{1}\right)=\frac{1}{2}, & F_{2}\left(e_{2}\right)\left(x_{2}\right)=\frac{1}{3}, & F_{2}\left(e_{2}\right)\left(x_{3}\right)=1, \\
F_{3}\left(e_{1}\right)\left(x_{1}\right)=\frac{1}{2}, & F_{3}\left(e_{1}\right)\left(x_{2}\right)=0, & F_{3}\left(e_{1}\right)\left(x_{3}\right)=\frac{1}{4}, \\
F_{3}\left(e_{2}\right)\left(x_{1}\right)=\frac{1}{3}, & F_{3}\left(e_{2}\right)\left(x_{2}\right)=\frac{1}{3}, & F_{3}\left(e_{2}\right)\left(x_{3}\right)=\frac{1}{4}, \\
F_{4}\left(e_{1}\right)\left(x_{1}\right)=1, & F_{4}\left(e_{1}\right)\left(x_{2}\right)=\frac{1}{3}, & F_{4}\left(e_{1}\right)\left(x_{3}\right)=\frac{1}{2}, \\
F_{4}\left(e_{2}\right)\left(x_{1}\right)=\frac{1}{2}, & F_{4}\left(e_{2}\right)\left(x_{2}\right)=\frac{1}{2}, & F_{4}\left(e_{2}\right)\left(x_{3}\right)=1 .
\end{array}
$$

Then $\tau$ defines a fuzzy soft topology on $X$ and hence $(X, \tau, E)$ is a fuzzy soft topological space over $X$. Then it can be easily seen that

$$
\begin{aligned}
& \tau_{e_{1}}=\left\{\underline{0}, \underline{1}, F_{1}\left(e_{1}\right), F_{2}\left(e_{1}\right), F_{3}\left(e_{1}\right), F_{4}\left(e_{1}\right)\right\}, \\
& \tau_{e_{2}}=\left\{\underline{0}, \underline{1}, F_{1}\left(e_{2}\right), F_{2}\left(e_{2}\right), F_{3}\left(e_{2}\right), F_{4}\left(e_{2}\right)\right\}
\end{aligned}
$$

are fuzzy topologies on $X$.

Now we show that the converse of the above proposition does not hold.

Example 18. Let $X=\left\{x_{1}, x_{2}\right\}$ and $E=\left\{e_{1}, e_{2}\right\}$. The fuzzy soft sets $F_{i}: E \rightarrow \widetilde{P}(X)$ on $X$, for $1 \leq i \leq 5$, are defined as follows:

$$
\begin{array}{ll}
F_{1}\left(e_{1}\right)\left(x_{1}\right)=\frac{1}{2}, & F_{1}\left(e_{1}\right)\left(x_{2}\right)=\frac{2}{3}, \\
F_{1}\left(e_{2}\right)\left(x_{1}\right)=\frac{1}{5}, & F_{1}\left(e_{2}\right)\left(x_{2}\right)=\frac{3}{5}, \\
F_{2}\left(e_{1}\right)\left(x_{1}\right)=\frac{1}{3}, & F_{2}\left(e_{1}\right)\left(x_{2}\right)=1,
\end{array}
$$

$$
\begin{array}{ll}
F_{2}\left(e_{2}\right)\left(x_{1}\right)=\frac{1}{7}, & F_{2}\left(e_{2}\right)\left(x_{2}\right)=\frac{4}{5}, \\
F_{3}\left(e_{1}\right)\left(x_{1}\right)=\frac{1}{3}, & F_{3}\left(e_{1}\right)\left(x_{2}\right)=\frac{2}{3}, \\
F_{3}\left(e_{2}\right)\left(x_{1}\right)=\frac{1}{7}, & F_{3}\left(e_{2}\right)\left(x_{2}\right)=\frac{3}{5}, \\
F_{4}\left(e_{1}\right)\left(x_{1}\right)=\frac{1}{2}, & F_{4}\left(e_{1}\right)\left(x_{2}\right)=1, \\
F_{4}\left(e_{2}\right)\left(x_{1}\right)=\frac{1}{5}, & F_{4}\left(e_{2}\right)\left(x_{2}\right)=\frac{4}{5}, \\
F_{5}\left(e_{1}\right)\left(x_{1}\right)=\frac{2}{3}, & F_{5}\left(e_{1}\right)\left(x_{2}\right)=1, \\
F_{5}\left(e_{2}\right)\left(x_{1}\right)=\frac{1}{10}, & F_{5}\left(e_{2}\right)\left(x_{2}\right)=\frac{2}{5} .
\end{array}
$$

Then

$$
\begin{aligned}
& \tau_{e_{1}}=\left\{\underline{0}, \underline{1}, F_{1}\left(e_{1}\right), F_{2}\left(e_{1}\right), F_{3}\left(e_{1}\right), F_{4}\left(e_{1}\right), F_{5}\left(e_{1}\right)\right\}, \\
& \tau_{e_{2}}=\left\{\underline{0}, \underline{1}, F_{1}\left(e_{2}\right), F_{2}\left(e_{2}\right), F_{3}\left(e_{2}\right), F_{4}\left(e_{2}\right), F_{5}\left(e_{2}\right)\right\}
\end{aligned}
$$

are fuzzy topologies on $X$. Here $\tau=\left\{\Phi, 1,\left(F_{1}, E\right),\left(F_{2}, E\right)\right.$, $\left.\left(F_{3}, E\right),\left(F_{4}, E\right),\left(F_{5}, E\right)\right\}$ is not a fuzzy soft topology on $X$ because

$$
\begin{array}{ll}
\left(F_{2} \vee F_{5}\right)\left(e_{1}\right)\left(x_{1}\right)=\frac{2}{3}, & \left(F_{2} \vee F_{5}\right)\left(e_{1}\right)\left(x_{2}\right)=1, \\
\left(F_{2} \vee F_{5}\right)\left(e_{2}\right)\left(x_{1}\right)=\frac{1}{7}, & \left(F_{2} \vee F_{5}\right)\left(e_{2}\right)\left(x_{2}\right)=\frac{4}{5},
\end{array}
$$

and so $\left(F_{2}, E\right) \vee\left(F_{5}, E\right) \notin \tau$.

Proposition 19. Let $\left(X, \tau_{1}, E\right)$ and $\left(X, \tau_{2}, E\right)$ be two fuzzy soft topological spaces over the same universe $X$, and then $\left(X, \tau_{1} \wedge\right.$ $\left.\tau_{2}, E\right)$ is a fuzzy soft topological spaces over $X$.

Proof. (1) $\Phi, 1$ belong to $\tau_{1} \wedge \tau_{2}$.

(2) Let $\left\{\left(F_{s}, E\right) \mid s \in S\right\}$ be a family of fuzzy soft sets in $\tau_{1} \wedge \tau_{2}$. Then $\left(F_{s}, E\right) \in \tau_{1}$ and $\left(F_{s}, E\right) \in \tau_{2}$, for all $s \in S$. Thus $\vee_{s \in S}\left(F_{s}, E\right) \in \tau_{1}$ and $\vee_{s \in S}\left(F_{s}, E\right) \in \tau_{2}$. This implies that $\vee_{s \in S}\left(F_{s}, E\right) \in \tau_{1} \wedge \tau_{2}$

(3) Let $(F, E),(G, E) \in \tau_{1} \wedge \tau_{2}$. Then $(F, E),(G, E) \in \tau_{1}$ and $(F, E),(G, E) \in \tau_{2}$. Since $(F, E) \wedge(G, E) \in \tau_{1}$ and $(F, E) \wedge$ $(G, E) \in \tau_{2}$, so $(F, E) \wedge(G, E) \in \tau_{1} \wedge \tau_{2}$.

Notice that $\tau_{1} \wedge \tau_{2}$ defines a fuzzy soft topology on $X$ and $\left(X, \tau_{1} \wedge \tau_{2}, E\right)$ is a fuzzy soft topological space over $X$.

Remark 20. The union of two fuzzy soft topologies on $X$ may not be a fuzzy soft topology on $X$.

Example 21. Let $X=\left\{x_{1}, x_{2}, x_{3}\right\}, E=\left\{e_{1}, e_{2}\right\}$ and $\tau_{1}=$ $\left\{\Phi, 1,\left(F_{1}, E\right),\left(F_{2}, E\right),\left(F_{3}, E\right),\left(F_{4}, E\right)\right\}, \tau_{2}=\left\{\Phi, 1,\left(G_{1}, E\right),\left(G_{2}\right.\right.$, $\left.E),\left(G_{3}, E\right),\left(G_{4}, E\right)\right\}$ be two soft topologies defined on $X$. The 
fuzzy soft sets $F_{i}, G_{i}: E \rightarrow \widetilde{P}(X)$ on $X$, for $1 \leq i \leq 4$, are defined as follows:

$$
\begin{aligned}
& F_{1}\left(e_{1}\right)\left(x_{1}\right)=\frac{1}{2}, \quad F_{1}\left(e_{1}\right)\left(x_{2}\right)=\frac{1}{3}, \quad F_{1}\left(e_{1}\right)\left(x_{3}\right)=\frac{1}{4}, \\
& F_{1}\left(e_{2}\right)\left(x_{1}\right)=0, \quad F_{1}\left(e_{2}\right)\left(x_{2}\right)=\frac{1}{2}, \quad F_{1}\left(e_{2}\right)\left(x_{3}\right)=1 \text {, } \\
& F_{2}\left(e_{1}\right)\left(x_{1}\right)=\frac{1}{5}, \quad F_{2}\left(e_{1}\right)\left(x_{2}\right)=\frac{1}{2}, \quad F_{2}\left(e_{1}\right)\left(x_{3}\right)=0 \text {, } \\
& F_{2}\left(e_{2}\right)\left(x_{1}\right)=\frac{1}{4}, \quad F_{2}\left(e_{2}\right)\left(x_{2}\right)=\frac{1}{2}, \quad F_{2}\left(e_{2}\right)\left(x_{3}\right)=0 \text {, } \\
& F_{3}\left(e_{1}\right)\left(x_{1}\right)=\frac{1}{2}, \quad F_{3}\left(e_{1}\right)\left(x_{2}\right)=\frac{1}{2}, \quad F_{3}\left(e_{1}\right)\left(x_{3}\right)=\frac{1}{4}, \\
& F_{3}\left(e_{2}\right)\left(x_{1}\right)=\frac{1}{4}, \quad F_{3}\left(e_{2}\right)\left(x_{2}\right)=\frac{1}{2}, \quad F_{3}\left(e_{2}\right)\left(x_{3}\right)=1 \text {, } \\
& F_{4}\left(e_{1}\right)\left(x_{1}\right)=\frac{1}{5}, \quad F_{4}\left(e_{1}\right)\left(x_{2}\right)=\frac{1}{3}, \quad F_{4}\left(e_{1}\right)\left(x_{3}\right)=0 \text {, } \\
& F_{4}\left(e_{2}\right)\left(x_{1}\right)=0, \quad F_{4}\left(e_{2}\right)\left(x_{2}\right)=\frac{1}{2}, \quad F_{4}\left(e_{2}\right)\left(x_{3}\right)=0, \\
& \left(G_{1}, E\right)=\left(F_{1}, E\right) \\
& G_{2}\left(e_{1}\right)\left(x_{1}\right)=0, \quad G_{2}\left(e_{1}\right)\left(x_{2}\right)=\frac{2}{3}, \quad G_{2}\left(e_{1}\right)\left(x_{3}\right)=\frac{1}{5}, \\
& G_{2}\left(e_{2}\right)\left(x_{1}\right)=1, \quad G_{2}\left(e_{2}\right)\left(x_{2}\right)=\frac{1}{7}, \quad G_{2}\left(e_{2}\right)\left(x_{3}\right)=\frac{2}{5}, \\
& G_{3}\left(e_{1}\right)\left(x_{1}\right)=\frac{1}{2}, \quad G_{3}\left(e_{1}\right)\left(x_{2}\right)=\frac{2}{3}, \quad G_{3}\left(e_{1}\right)\left(x_{3}\right)=\frac{1}{4}, \\
& G_{3}\left(e_{2}\right)\left(x_{1}\right)=1, \quad G_{3}\left(e_{2}\right)\left(x_{2}\right)=\frac{1}{2}, \quad G_{3}\left(e_{2}\right)\left(x_{3}\right)=1, \\
& G_{4}\left(e_{1}\right)\left(x_{1}\right)=0, \quad G_{4}\left(e_{1}\right)\left(x_{2}\right)=\frac{1}{3}, \quad G_{4}\left(e_{1}\right)\left(x_{3}\right)=\frac{1}{5}, \\
& G_{4}\left(e_{2}\right)\left(x_{1}\right)=0, \quad G_{4}\left(e_{2}\right)\left(x_{2}\right)=\frac{1}{7}, \quad G_{4}\left(e_{2}\right)\left(x_{2}\right)=\frac{2}{5} \text {. }
\end{aligned}
$$

We define $\tau=\tau_{1} \vee \tau_{2}=\left\{\Phi, 1,\left(F_{1}, E\right),\left(F_{2}, E\right),\left(F_{3}, E\right),\left(F_{4}, E\right)\right.$, $\left.\left(G_{2}, E\right),\left(G_{3}, E\right),\left(G_{4}, E\right)\right\}$.

If we take

$$
\begin{array}{ll}
\left(F_{2} \vee G_{4}\right)\left(e_{1}\right)\left(x_{1}\right)=\frac{1}{5}, & \left(F_{2} \vee G_{4}\right)\left(e_{1}\right)\left(x_{2}\right)=\frac{1}{2} \\
\left(F_{2} \vee G_{4}\right)\left(e_{1}\right)\left(x_{3}\right)=\frac{1}{5}, & \left(F_{2} \vee G_{4}\right)\left(e_{2}\right)\left(x_{1}\right)=\frac{1}{4} \\
\left(F_{2} \vee G_{4}\right)\left(e_{2}\right)\left(x_{2}\right)=\frac{1}{2}, & \left(F_{2} \vee G_{4}\right)\left(e_{2}\right)\left(x_{3}\right)=\frac{2}{5}
\end{array}
$$

then $\left(F_{2}, E\right) \vee\left(G_{4}, E\right) \notin \tau$.

Definition 22. Let $(X, \tau, E)$ be a fuzzy soft topological space over $X$ and $(F, E)$ be a fuzzy soft set over $X$. Then we associate with $(F, E)$ a fuzzy soft set over $X$, denoted by $(\bar{F}, E)$ and defined as $\bar{F}(\alpha)=\overline{F(\alpha)}$, where $\overline{F(\alpha)}$ is the closure of fuzzy set $F(\alpha)$ in $\tau_{\alpha}$ for each $\alpha \in E$.
Proposition 23. Let $(X, \tau, E)$ be a fuzzy soft topological space over $X$ and $(F, E)$ be a fuzzy soft set over $X$. Then $(\bar{F}, E) \widetilde{\subset} \overline{(F, E)}$.

Proof. For any $\alpha \in E, \overline{F(\alpha)}$ is the smallest closed set in $\left(X, \tau_{\alpha}\right)$ which contains $F(\alpha)$. So $\overline{(F, E)}=(H, E)$ and then $H(\alpha)$ is also a closed set in $\left(X, \tau_{\alpha}\right)$ containing $F(\alpha)$. This implies that $\bar{F}(\alpha) \leq H(\alpha)$. Thus $(\bar{F}, E) \widetilde{\subset} \overline{(F, E)}$.

Corollary 24. Let $(X, \tau, E)$ be a fuzzy soft topological space over $X$ and $(F, E)$ be a fuzzy soft set over $X$. Then $(\bar{F}, E)=$ $\overline{(F, E)}$ if and only if $(\bar{F}, E)^{\prime} \in \tau$.

Proof. If $(\bar{F}, E)=\overline{(F, E)}$, then $(\bar{F}, E)$ is fuzzy soft closed set and so $(\bar{F}, E)^{\prime} \in \tau$. Conversely if $(\bar{F}, E)^{\prime} \in \tau$, then $(\bar{F}, E)$ is a fuzzy soft closed set containing $(F, E)$. By the definition of fuzzy soft closure of $(F, E),(\overline{F, E}) \widetilde{C}(\bar{F}, E)$. By proposition $(\bar{F}, E) \tilde{\mathcal{C}} \overline{(F, E)}$. Thus $(\bar{F}, E)=\overline{(F, E)}$.

Example 25. Let $X=\left\{x_{1}, x_{2}\right\}, E=\left\{e_{1}, e_{2}\right\}$, and $\tau=$ $\left\{\Phi, 1,\left(F_{1}, E\right),\left(F_{2}, E\right), \ldots,\left(F_{5}, E\right)\right\}$, where

$$
\begin{array}{ll}
F_{1}\left(e_{1}\right)\left(x_{1}\right)=\frac{1}{2}, & F_{1}\left(e_{1}\right)\left(x_{2}\right)=\frac{2}{3}, \\
F_{1}\left(e_{2}\right)\left(x_{1}\right)=0, & F_{1}\left(e_{2}\right)\left(x_{2}\right)=\frac{4}{5}, \\
F_{2}\left(e_{1}\right)\left(x_{1}\right)=\frac{2}{3}, & F_{2}\left(e_{1}\right)\left(x_{2}\right)=1, \\
F_{2}\left(e_{2}\right)\left(x_{1}\right)=0, & F_{2}\left(e_{2}\right)\left(x_{2}\right)=\frac{1}{3}, \\
F_{3}\left(e_{1}\right)\left(x_{1}\right)=\frac{1}{2}, & F_{3}\left(e_{1}\right)\left(x_{2}\right)=\frac{2}{3}, \\
F_{3}\left(e_{2}\right)\left(x_{1}\right)=0, & F_{3}\left(e_{2}\right)\left(x_{2}\right)=\frac{1}{3}, \\
F_{4}\left(e_{1}\right)\left(x_{1}\right)=\frac{2}{3}, & F_{4}\left(e_{1}\right)\left(x_{2}\right)=1, \\
F_{4}\left(e_{2}\right)\left(x_{1}\right)=0, & F_{4}\left(e_{2}\right)\left(x_{2}\right)=\frac{4}{5}, \\
F_{5}\left(e_{1}\right)\left(x_{1}\right)=\frac{5}{6}, & F_{5}\left(e_{1}\right)\left(x_{2}\right)=1, \\
F_{5}\left(e_{2}\right)\left(x_{1}\right)=\frac{1}{10}, & F_{5}\left(e_{2}\right)\left(x_{2}\right)=\frac{9}{10},
\end{array}
$$

and then $(X, \tau, E)$ is a fuzzy soft topological space over $X$. If $(F, E)$ is defined as follows:

$$
\begin{array}{ll}
F\left(e_{1}\right)\left(x_{1}\right)=\frac{1}{3}, & F\left(e_{1}\right)\left(x_{2}\right)=\frac{1}{4}, \\
F\left(e_{2}\right)\left(x_{1}\right)=\frac{1}{2}, & F\left(e_{2}\right)\left(x_{2}\right)=0
\end{array}
$$

then

$$
\overline{(F, E)}=\widetilde{X} \wedge\left(F_{1}, E\right)^{\prime} \wedge\left(F_{3}, E\right)^{\prime}=\left(F_{1}, E\right)^{\prime} .
$$


From the simple calculation,

$$
\begin{array}{ll}
\bar{F}\left(e_{1}\right)\left(x_{1}\right)=\frac{1}{2}, & \bar{F}\left(e_{1}\right)\left(x_{2}\right)=\frac{1}{3}, \\
\bar{F}\left(e_{2}\right)\left(x_{1}\right)=\frac{9}{10}, & \bar{F}\left(e_{2}\right)\left(x_{2}\right)=\frac{1}{10}
\end{array}
$$

are obtained. It is clear that

$$
(\bar{F}, E) \neq \overline{(F, E)}
$$

Definition 26. Let $(X, \tau, E)$ be a fuzzy soft topological space over $X$ and $(F, E)$ be a fuzzy soft set over $X$. Then we associate with $(F, E)$ a fuzzy soft set over $X$, denoted by $\left(F^{\circ}, E\right)$ and defined as $F^{\circ}(\alpha)=(F(\alpha))^{\circ}$, where $(F(\alpha))^{\circ}$ is an interior of fuzzy set $F(\alpha)$ in $\tau_{\alpha}$, for each $\alpha \in E$.

Proposition 27. Let $(X, \tau, E)$ be a fuzzy soft topological space over $X$ and $(F, E)$ be a fuzzy soft set over $X$. Then ( $F$, $E)^{\circ} \tilde{\subset}\left(F^{\circ}, E\right)$.

Proof. For each $\alpha \in E, F^{\circ}(\alpha)$ is the biggest open fuzzy set in $\left(X, \tau_{\alpha}\right)$ which is belonging $F(\alpha)$. Since $(F, E)^{\circ} \widetilde{C}(F, E)$, then $(F, E)^{\circ} \stackrel{\alpha}{\widetilde{C}}\left(F^{\circ}, E\right)$ is satisfied.

Corollary 28. Let $(X, \tau, E)$ be a fuzzy soft topological space over $X$ and $(F, E)$ be a fuzzy soft set over $X$. Then $(F, E)^{\circ}=\left(F^{\circ}\right.$, $E)$ if and only if $\left(F^{\circ}, E\right)^{\prime}$ is a fuzzy soft closed set.

Proof. If $(F, E)^{\circ}=\left(F^{\circ}, E\right)$, then $\left(F^{\circ}, E\right)$ is a fuzzy soft open set, and so $\left(F^{\circ}, E\right)^{\prime}$ is a fuzzy soft closed set. Conversely if $\left(F^{\circ}, E\right)^{\prime}$ is a fuzzy soft closed set, then $\left(F^{\circ}, E\right)$ is a fuzzy soft open set and $\left(F^{\circ}, E\right) \widetilde{C}(F, E)$. By the definition of fuzzy soft interior of $(F, E),\left(F^{\circ}, E\right) \widetilde{C}(F, E)^{\circ}$ is satisfied. Then by Proposition 27, since $(F, E)^{\circ} \widetilde{C}\left(F^{\circ}, E\right),(F, E)^{\circ}=\left(F^{\circ}, E\right)$ is obtained.

Example 29. Let $X=\left\{x_{1}, x_{2}\right\}, E=\left\{e_{1}, e_{2}\right\}$ and $\tau=\left\{\Phi, 1,\left(F_{1}\right.\right.$, $\left.E),\left(F_{2}, E\right), \ldots,\left(F_{5}, E\right)\right\}$ be a fuzzy soft topology over $X$, and the fuzzy soft sets are defined as follows:

$$
\begin{array}{ll}
F_{1}\left(e_{1}\right)\left(x_{1}\right)=\frac{1}{2}, & F_{1}\left(e_{1}\right)\left(x_{2}\right)=\frac{2}{3}, \\
F_{1}\left(e_{2}\right)\left(x_{1}\right)=0, & F_{1}\left(e_{2}\right)\left(x_{2}\right)=\frac{4}{5}, \\
F_{2}\left(e_{1}\right)\left(x_{1}\right)=\frac{2}{3}, & F_{2}\left(e_{1}\right)\left(x_{2}\right)=1, \\
F_{2}\left(e_{2}\right)\left(x_{1}\right)=0, & F_{2}\left(e_{2}\right)\left(x_{2}\right)=\frac{1}{3}, \\
F_{3}\left(e_{1}\right)\left(x_{1}\right)=\frac{1}{2}, & F_{3}\left(e_{1}\right)\left(x_{2}\right)=\frac{2}{3}, \\
F_{3}\left(e_{2}\right)\left(x_{1}\right)=0, & F_{3}\left(e_{2}\right)\left(x_{2}\right)=\frac{1}{3}, \\
F_{4}\left(e_{1}\right)\left(x_{1}\right)=\frac{2}{3}, & F_{4}\left(e_{1}\right)\left(x_{2}\right)=1,
\end{array}
$$

$$
\begin{array}{ll}
F_{4}\left(e_{2}\right)\left(x_{1}\right)=0, & F_{4}\left(e_{2}\right)\left(x_{2}\right)=\frac{4}{5}, \\
F_{5}\left(e_{1}\right)\left(x_{1}\right)=\frac{5}{6}, & F_{5}\left(e_{1}\right)\left(x_{2}\right)=1, \\
F_{5}\left(e_{2}\right)\left(x_{1}\right)=\frac{1}{10}, & F_{5}\left(e_{2}\right)\left(x_{2}\right)=\frac{9}{10} .
\end{array}
$$

Now we define fuzzy soft set $(F, E)$ as follows:

$$
\begin{aligned}
& F\left(e_{1}\right)\left(x_{1}\right)=1, \quad F\left(e_{1}\right)\left(x_{2}\right)=\frac{5}{6}, \\
& F\left(e_{2}\right)\left(x_{1}\right)=\frac{3}{20}, \quad F\left(e_{2}\right)\left(x_{2}\right)=\frac{19}{20} .
\end{aligned}
$$

Then

$$
(F, E)^{\circ}=\left(F_{1}, E\right) \vee\left(F_{3}, E\right)=(H, E) .
$$

Here

$$
\begin{array}{ll}
H\left(e_{1}\right)\left(x_{1}\right)=\frac{1}{2}, & H\left(e_{1}\right)\left(x_{2}\right)=\frac{2}{3}, \\
H\left(e_{2}\right)\left(x_{1}\right)=0, & H\left(e_{2}\right)\left(x_{2}\right)=\frac{4}{5} \\
F^{\circ}\left(e_{1}\right)\left(x_{1}\right)=\frac{1}{2}, & F^{\circ}\left(e_{1}\right)\left(x_{2}\right)=\frac{2}{3}, \\
F^{\circ}\left(e_{2}\right)\left(x_{1}\right)=\frac{1}{10}, & F^{\circ}\left(e_{2}\right)\left(x_{2}\right)=\frac{9}{10} .
\end{array}
$$

Clearly, $(F, E)^{\circ} \neq\left(F^{\circ}, E\right)$.

Definition 30. Let $(F, E)$ and $(G, E)$ be two fuzzy soft sets. The difference of two soft sets $(F, E)$ and $(G, E)$ over $X$, denoted by $(F, E) \backslash(G, E)$, is defined as $(F, E) \backslash(G, E)=(F, E) \wedge(G, E)^{\prime}$.

Definition 31. Let $(X, \tau, E)$ be a fuzzy soft topological space over $X$ and $(F, E)$ be a fuzzy soft set over $X$. Then the fuzzy soft boundary of $(F, E)$, denoted by $\partial(F, E)$, is defined as $\partial(F, E)=$ $\overline{(F, E)} \wedge \overline{(F, E)^{\prime}}$.

Theorem 32. Let $(X, \tau, E)$ be a fuzzy soft topological space over $X$ and $(F, E)$ be a fuzzy soft set over $X$. Then

(1) $(F, E)^{\circ}=(F, E) \backslash \partial(F, E)$,

(2) $\overline{(F, E)}=(F, E) \vee \partial(F, E)$,

(3) $(F, E) \in \tau \Leftrightarrow \partial(F, E)=\overline{(F, E)} \backslash(F, E)$,

(4) $(F, E)^{\prime} \in \tau \Leftrightarrow \partial(F, E)=(F, E) \backslash(F, E)^{\circ}$. 
Proof. (1) Let $(F, E)$ be a fuzzy soft set over $X$. Then we have

$$
\begin{aligned}
(F, E) \backslash \partial(F, E) \\
=(F, E) \backslash\left(\overline{(F, E)} \wedge \overline{(F, E)^{\prime}}\right) \\
=(F, E) \wedge\left(\overline{(F, E)} \wedge \overline{(F, E)^{\prime}}\right)^{\prime} \\
=(F, E) \wedge\left(\overline{(F, E)} \vee^{\prime} \overline{(F, E)^{\prime}}\right) \\
=(F, E) \wedge(F, E)^{\circ}=(F, E)^{\circ} .
\end{aligned}
$$

(2) From Definition 31, it follows that

$$
\begin{aligned}
(F, E) & \vee\left(\overline{(F, E)} \wedge \overline{(F, E)^{\prime}}\right) \\
& =[(F, E) \vee \overline{(F, E)}] \wedge\left[(F, E) \vee \overline{(F, E)^{\prime}}\right] \\
& =\overline{(F, E)} \wedge \widetilde{X}=\overline{(F, E)} .
\end{aligned}
$$

(3) Suppose that $(F, E) \in \tau$. Then $(F, E)=(F, E)^{\circ}$. Hence

$$
\begin{aligned}
\partial(F, E)= & \overline{(F, E)} \wedge \overline{(F, E)^{\prime}} \\
& =\overline{(F, E)} \backslash \overline{(F, E)^{\prime}}=\overline{(F, E)} \backslash(F, E)^{\circ} \\
& =\overline{(F, E)} \backslash(F, E) \\
& \partial(F, E)=\overline{(F, E)} \backslash(F, E)
\end{aligned}
$$

is obtained. Conversely, suppose that $\partial(F, E)=\overline{(F, E)} \backslash(F, E)$. From Definition 31,

$$
\begin{aligned}
\partial(F, E) & =\overline{(F, E)} \wedge \overline{(F, E)^{\prime}} \\
& =\overline{(F, E)} \backslash \overline{(F, E)^{\prime}} \\
& =\overline{(F, E)} \backslash(F, E)^{\circ} .
\end{aligned}
$$

This implies that $(F, E) \in \tau$. Hence

(4) Let $(F, E)^{\prime} \in \tau$. Then $(F, E)$ is a fuzzy soft closed set.

$$
\partial(F, E)=(F, E) \wedge \overline{(F, E)^{\prime}}=(F, E) \backslash(F, E)^{\circ}
$$

is obtained. Conversely, $\partial(F, E)=(F, E) \backslash(F, E)^{\circ}$. From Definition 31,

$$
\partial(F, E)=\overline{(F, E)} \wedge \overline{(F, E)^{\prime}}=\overline{(F, E)} \backslash(F, E)^{\circ} .
$$

It is clear that $(F, E)=\overline{(F, E)}$. This implies that $(F, E)^{\prime} \in \tau$.

\section{Fuzzy Soft Continuous Mappings}

Definition 33 (see [21]). Let $(X, \tau, E)$ and $\left(Y, \tau^{\prime}, E\right)$ be two fuzzy soft topological spaces and $f:(X, \tau, E) \rightarrow\left(Y, \tau^{\prime}, E\right)$ be a mapping. For each $(G, E) \in \tau^{\prime}$, if $f^{-1}(G, E) \in \tau$, then $f:(X, \tau, E) \rightarrow\left(Y, \tau^{\prime}, E\right)$ is said to be fuzzy soft continuous mapping of fuzzy soft topological spaces.

Let us investigate some properties of fuzzy soft continuous mappings.
Proposition 34. If mapping $f:(X, \tau, E) \rightarrow\left(Y, \tau^{\prime}, E\right)$ is a fuzzy soft continuous mapping, then, for each $\alpha \in E, f:\left(X, \tau_{\alpha}\right)$ $\rightarrow\left(Y, \tau_{\alpha}^{\prime}\right)$ is a fuzzy continuous mapping.

Proof. Let $U \in \tau_{\alpha}^{\prime}$. Then there exists a fuzzy soft open set $(G, E)$ over $Y$ such that $U=G(\alpha)$. Since $f:(X, \tau, E) \rightarrow(Y$, $\left.\tau^{\prime}, E\right)$ is a fuzzy soft continuous mapping, $f^{-1}(G, E)$ is a fuzzy soft open set over $X$ and $f^{-1}(G, E)(\alpha)=f^{-1}(G(\alpha))=f^{-1}(U)$ is a fuzzy open set. This implies that $f$ is a fuzzy continuous mapping.

Now we give an example to show that the converse of above proposition does not hold.

Example 35. Let $X=\left\{x_{1}, x_{2}, x_{3}\right\}, Y=\left\{y_{1}, y_{2}, y_{3}\right\}$ and $E=$ $\left\{e_{1}, e_{2}\right\}$.

Here $\left\{\left(F_{i}, E\right): 1 \leq i \leq 9\right\}$ are fuzzy soft sets over $X$ and $\left(G_{1}, E\right),\left(G_{2}, E\right)$ are fuzzy soft sets over $Y$, defined as follows:

$$
\begin{aligned}
& F_{1}\left(e_{1}\right)\left(x_{1}\right)=\frac{2}{3}, \quad F_{1}\left(e_{1}\right)\left(x_{2}\right)=\frac{1}{2}, \\
& F_{1}\left(e_{1}\right)\left(x_{3}\right)=\frac{1}{2}, \quad F_{1}\left(e_{2}\right)\left(x_{1}\right)=\frac{5}{7}, \\
& F_{1}\left(e_{2}\right)\left(x_{2}\right)=\frac{1}{5}, \quad F_{1}\left(e_{2}\right)\left(x_{3}\right)=\frac{1}{5}, \\
& F_{2}\left(e_{1}\right)\left(x_{1}\right)=\frac{1}{4}, \quad F_{2}\left(e_{1}\right)\left(x_{2}\right)=\frac{1}{4}, \\
& F_{2}\left(e_{1}\right)\left(x_{3}\right)=\frac{1}{4}, \quad F_{2}\left(e_{2}\right)\left(x_{1}\right)=0 \text {, } \\
& F_{2}\left(e_{2}\right)\left(x_{2}\right)=1, \quad F_{2}\left(e_{2}\right)\left(x_{3}\right)=\frac{1}{2}, \\
& F_{3}\left(e_{1}\right)\left(x_{1}\right)=\frac{1}{2}, \quad F_{3}\left(e_{1}\right)\left(x_{2}\right)=\frac{1}{5}, \\
& F_{3}\left(e_{1}\right)\left(x_{3}\right)=\frac{1}{5}, \quad F_{3}\left(e_{2}\right)\left(x_{1}\right)=\frac{1}{2}, \\
& F_{3}\left(e_{2}\right)\left(x_{2}\right)=0, \quad F_{3}\left(e_{2}\right)\left(x_{3}\right)=0, \\
& F_{4}\left(e_{1}\right)\left(x_{1}\right)=\frac{1}{4}, \quad F_{4}\left(e_{1}\right)\left(x_{2}\right)=\frac{1}{4}, \\
& F_{4}\left(e_{1}\right)\left(x_{3}\right)=\frac{1}{4}, \quad F_{4}\left(e_{2}\right)\left(x_{1}\right)=0, \\
& F_{4}\left(e_{2}\right)\left(x_{2}\right)=\frac{1}{5}, \quad F_{4}\left(e_{2}\right)\left(x_{3}\right)=\frac{1}{5}, \\
& F_{5}\left(e_{1}\right)\left(x_{1}\right)=\frac{1}{2}, \quad F_{5}\left(e_{1}\right)\left(x_{2}\right)=\frac{1}{5}, \\
& F_{5}\left(e_{1}\right)\left(x_{3}\right)=\frac{1}{5}, \quad F_{5}\left(e_{2}\right)\left(x_{1}\right)=\frac{1}{2} \text {, } \\
& F_{5}\left(e_{2}\right)\left(x_{2}\right)=0, \quad F_{5}\left(e_{2}\right)\left(x_{3}\right)=0 \text {, }
\end{aligned}
$$




$$
\begin{aligned}
& F_{6}\left(e_{1}\right)\left(x_{1}\right)=\frac{1}{4}, \quad F_{6}\left(e_{1}\right)\left(x_{2}\right)=\frac{1}{5}, \\
& F_{6}\left(e_{1}\right)\left(x_{3}\right)=\frac{1}{5}, \quad F_{6}\left(e_{2}\right)\left(x_{1}\right)=0, \\
& F_{6}\left(e_{2}\right)\left(x_{2}\right)=0, \quad F_{6}\left(e_{2}\right)\left(x_{3}\right)=0, \\
& F_{7}\left(e_{1}\right)\left(x_{1}\right)=\frac{1}{2}, \quad F_{7}\left(e_{1}\right)\left(x_{2}\right)=\frac{1}{4}, \\
& F_{7}\left(e_{1}\right)\left(x_{3}\right)=\frac{1}{4}, \quad F_{7}\left(e_{2}\right)\left(x_{1}\right)=\frac{1}{2}, \\
& F_{7}\left(e_{2}\right)\left(x_{2}\right)=1, \quad F_{7}\left(e_{2}\right)\left(x_{3}\right)=\frac{1}{2}, \\
& F_{8}\left(e_{1}\right)\left(x_{1}\right)=\frac{2}{3}, \quad F_{8}\left(e_{1}\right)\left(x_{2}\right)=\frac{1}{2}, \\
& F_{8}\left(e_{1}\right)\left(x_{3}\right)=\frac{1}{2}, \quad F_{8}\left(e_{2}\right)\left(x_{1}\right)=\frac{5}{7}, \\
& F_{8}\left(e_{2}\right)\left(x_{2}\right)=1, \quad F_{8}\left(e_{2}\right)\left(x_{3}\right)=\frac{1}{2} \\
& F_{9}\left(e_{1}\right)\left(x_{1}\right)=\frac{1}{2}, \quad F_{9}\left(e_{1}\right)\left(x_{2}\right)=\frac{1}{4}, \\
& F_{9}\left(e_{1}\right)\left(x_{3}\right)=\frac{1}{4}, \quad F_{9}\left(e_{2}\right)\left(x_{1}\right)=\frac{1}{2}, \\
& F_{9}\left(e_{2}\right)\left(x_{2}\right)=\frac{1}{5}, \quad F_{9}\left(e_{2}\right)\left(x_{3}\right)=\frac{1}{5}, \\
& G_{1}\left(e_{1}\right)\left(y_{1}\right)=\frac{1}{2}, \quad G_{1}\left(e_{1}\right)\left(y_{2}\right)=\frac{2}{3}, \\
& G_{1}\left(e_{1}\right)\left(y_{3}\right)=\frac{1}{4}, \quad G_{1}\left(e_{2}\right)\left(y_{1}\right)=\frac{1}{5}, \\
& G_{1}\left(e_{2}\right)\left(y_{2}\right)=\frac{5}{7}, \quad G_{1}\left(e_{2}\right)\left(y_{3}\right)=1, \\
& G_{2}\left(e_{1}\right)\left(y_{1}\right)=\frac{1}{4}, \quad G_{2}\left(e_{1}\right)\left(y_{2}\right)=\frac{1}{4}, \\
& G_{2}\left(e_{1}\right)\left(y_{3}\right)=\frac{1}{4}, \quad G_{2}\left(e_{2}\right)\left(y_{1}\right)=0, \\
& G_{2}\left(e_{2}\right)\left(y_{2}\right)=\frac{1}{2}, \quad G_{2}\left(e_{2}\right)\left(y_{3}\right)=\frac{1}{3} .
\end{aligned}
$$

If we get the mapping $f: X \rightarrow Y$ defined as

$$
f\left(x_{1}\right)=y_{2}, \quad f\left(x_{2}\right)=f\left(x_{3}\right)=y_{1},
$$

then $f$ is not a fuzzy soft continuous mapping. Since

$$
\begin{aligned}
& f^{-1}\left(G_{2}\right)\left(e_{1}\right)\left(x_{1}\right)=G_{2}\left(e_{1}\right)\left(f\left(x_{1}\right)\right)=G_{2}\left(e_{1}\right)\left(y_{2}\right)=\frac{1}{4}, \\
& f^{-1}\left(G_{2}\right)\left(e_{1}\right)\left(x_{2}\right)=G_{2}\left(e_{1}\right)\left(y_{1}\right)=\frac{1}{4}, \\
& f^{-1}\left(G_{2}\right)\left(e_{1}\right)\left(x_{3}\right)=\frac{1}{4},
\end{aligned}
$$

$$
\begin{aligned}
& f^{-1}\left(G_{2}\right)\left(e_{2}\right)\left(x_{1}\right)=G_{2}\left(e_{2}\right)\left(y_{2}\right)=\frac{1}{2}, \\
& f^{-1}\left(G_{2}\right)\left(e_{2}\right)\left(x_{2}\right)=G_{2}\left(e_{2}\right)\left(y_{1}\right)=0, \\
& f^{-1}\left(G_{2}\right)\left(e_{2}\right)\left(x_{3}\right)=0,
\end{aligned}
$$

we have $f^{-1}\left(G_{2}, E\right) \notin \tau$. Also,

$$
\begin{aligned}
& \tau_{e_{1}}=\left\{\underline{1}, \underline{0}, F_{1}\left(e_{1}\right), F_{2}\left(e_{1}\right), F_{3}\left(e_{1}\right),\right.\left.F_{6}\left(e_{1}\right), F_{7}\left(e_{1}\right), F_{8}\left(e_{1}\right)\right\} \\
& \tau_{e_{2}}=\left\{\underline{1}, \underline{0}, F_{1}\left(e_{2}\right), F_{2}\left(e_{2}\right), F_{3}\left(e_{2}\right),\right. \\
& \\
&\left.F_{4}\left(e_{2}\right), F_{7}\left(e_{2}\right), F_{8}\left(e_{2}\right), F_{9}\left(e_{2}\right)\right\} \\
& \tau_{e_{1}}^{\prime}=\left\{\underline{1}, \underline{0}, G_{1}\left(e_{1}\right), G_{2}\left(e_{1}\right)\right\} \\
& \tau_{e_{2}}^{\prime}=\left\{\underline{1}, \underline{0}, G_{1}\left(e_{2}\right), G_{2}\left(e_{2}\right)\right\} .
\end{aligned}
$$

The mapping $f:\left(X, \tau_{e_{1}}\right) \rightarrow\left(Y, \tau_{e_{1}}^{\prime}\right)$ is a fuzzy continuous mapping. Because

$$
\begin{aligned}
& f^{-1}\left(G_{1}\left(e_{1}\right)\right)\left(x_{1}\right)=G_{1}\left(e_{1}\right)\left(f\left(x_{1}\right)\right)=G_{1}\left(e_{1}\right)\left(y_{2}\right)=\frac{2}{3}, \\
& f^{-1}\left(G_{1}\left(e_{1}\right)\right)\left(x_{2}\right)=G_{1}\left(e_{1}\right)\left(y_{1}\right)=\frac{1}{2}, \\
& f^{-1}\left(G_{1}\left(e_{1}\right)\right)\left(x_{3}\right)=\frac{1}{2}, \\
& f^{-1}\left(G_{2}\left(e_{1}\right)\right)\left(x_{1}\right)=G_{2}\left(e_{1}\right)\left(y_{2}\right)=\frac{1}{4}, \\
& f^{-1}\left(G_{2}\left(e_{1}\right)\right)\left(x_{2}\right)=G_{2}\left(e_{1}\right)\left(y_{1}\right)=\frac{1}{4}, \\
& f^{-1}\left(G_{2}\left(e_{1}\right)\right)\left(x_{3}\right)=\frac{1}{4},
\end{aligned}
$$

hence, $f^{-1}\left(G_{1}\left(e_{1}\right)\right)=F_{1}\left(e_{1}\right), f^{-1}\left(G_{2}\left(e_{1}\right)\right)=F_{2}\left(e_{1}\right)$ is obtained.

Similarly, the mapping $f:\left(X, \tau_{e_{2}}\right) \rightarrow\left(Y, \tau_{e_{2}}^{\prime}\right)$ is a fuzzy continuous mapping. Because

$$
\begin{aligned}
& f^{-1}\left(G_{1}\left(e_{2}\right)\right)\left(x_{1}\right)=G_{1}\left(e_{2}\right)\left(y_{2}\right)=\frac{5}{7}, \\
& f^{-1}\left(G_{1}\left(e_{2}\right)\right)\left(x_{2}\right)=G_{1}\left(e_{2}\right)\left(y_{1}\right)=\frac{1}{5}, \\
& f^{-1}\left(G_{1}\left(e_{2}\right)\right)\left(x_{3}\right)=\frac{1}{5}, \\
& f^{-1}\left(G_{2}\left(e_{2}\right)\right)\left(x_{1}\right)=G_{2}\left(e_{2}\right)\left(y_{2}\right)=\frac{1}{2}, \\
& f^{-1}\left(G_{2}\left(e_{2}\right)\right)\left(x_{2}\right)=G_{2}\left(e_{2}\right)\left(y_{1}\right)=0, \\
& f^{-1}\left(G_{2}\left(e_{2}\right)\right)\left(x_{3}\right)=0,
\end{aligned}
$$

thus $f^{-1}\left(G_{1}\left(e_{2}\right)\right)=F_{1}\left(e_{2}\right), f^{-1}\left(G_{2}\left(e_{2}\right)\right)=F_{3}\left(e_{2}\right)$ is obtained. 
Definition 36. Let $(X, \tau, E)$ and $\left(Y, \tau^{\prime}, E\right)$ be two fuzzy soft topological spaces and $f: X \rightarrow Y$ be a mapping.

(a) If the image $f((F, E))$ of each fuzzy soft open set $(F, E)$ over $X$ is a fuzzy soft open set in $Y$, then $f$ is said to be fuzzy soft open mapping.

(b) If the image $f((H, E))$ of each fuzzy soft closed set $(H, E)$ over $X$ is a fuzzy soft closed set in $Y$, then $f$ is said to be fuzzy soft closed mapping.

Proposition 37. If $f:(X, \tau, E) \rightarrow\left(Y, \tau^{\prime}, E\right)$ is a fuzzy soft open (closed), then, for each $\alpha \in E, f:\left(X, \tau_{\alpha}\right) \rightarrow\left(Y, \tau_{\alpha}^{\prime}\right)$ is fuzzy open (closed) mapping.

Proof. The proof is straightforward.

Theorem 38. Let $(X, \tau, E)$ and $\left(Y, \tau^{\prime}, E\right)$ be two fuzzy soft topological spaces and $f: X \rightarrow Y$ be a mapping.

(a) $f$ is a fuzzy soft open mapping if and only if, for each fuzzy soft set $(F, E)$ over $X, f\left((F, E)^{\circ}\right) \widetilde{C}(f(F, E))^{\circ}$ is satisfied.

(b) $f$ is a fuzzy soft closed mapping if and only if for each fuzzy soft set $(F, E)$ over $X, \overline{(f(F, E))} \widetilde{c} f(\overline{(F, E)})$ is satisfied.

Proof. (a) Let $f$ be a fuzzy soft open mapping and $(F, E)$ be a fuzzy soft set over $X .(F, E)^{\circ}$ is a fuzzy soft open set and $(F, E)^{\circ} \tilde{C}(F, E)$. Since $f$ is a fuzzy soft open mapping, $f\left((F, E)^{\circ}\right)$ is a fuzzy soft open set in $Y$. Then $f\left((F, E)^{\circ}\right) \tilde{\subset} f((F, E))$. Thus $f\left((F, E)^{\circ}\right) \widetilde{\subset} f((F, E))^{\circ}$ is obtained.

Conversely, let $(F, E)$ be any fuzzy soft open set over $X$. Then $(F, E)=(F, E)^{\circ}$. From the condition of theorem, we have $f\left((F, E)^{\circ}\right) \widetilde{c} f((F, E))^{\circ}$. Then $f((F, E))=f\left((F, E)^{\circ}\right)$ $\widetilde{C}(f(F, E))^{\circ} \widetilde{\subset} f((F, E))$. This implies that $f((F, E))=$ $(f(F, E))^{\circ}$. This completes the proof.

(b) Let $f$ be a fuzzy soft closed mapping and $(F, E)$ be any fuzzy soft set over $X$. Since $f$ is a fuzzy soft closed mapping, $f(\overline{(F, E)})$ is a fuzzy soft closed set over $Y$ and $f((F, E)) \widetilde{\subset} f(\overline{(F, E)})$. Thus $\overline{(f(F, E))} \widetilde{\subset} f(\overline{(F, E)})$ is obtained.

Conversely, let $(F, E)$ be any fuzzy soft closed set over $X$. From the condition of theorem, $\overline{(f(F, E))} \widetilde{\subset} f(\overline{(F, E)})=$ $f((F, E)) \widetilde{C} \overline{(f(F, E))}$. This means that $\overline{(f(F, E))}=f((F, E))$. This completes the proof.

Note that the concepts of fuzzy soft continuous, fuzzy soft open, and fuzzy soft closed mappings are all independent of each other.

Example 39. Let $E=\left\{e_{1}, e_{2}\right\}, X=\left\{x_{1}, x_{2}\right\}$, and $Y=\left\{y_{1}, y_{2}\right\}$. We define the mapping $f: X \rightarrow Y$ as $f\left(x_{1}\right)=y_{1}, f\left(x_{2}\right)=$ $y_{1}$.

Here the fuzzy soft sets are defined as follows:

$$
\begin{array}{ll}
F_{1}\left(e_{1}\right)\left(x_{1}\right)=\frac{1}{3}, & F_{1}\left(e_{1}\right)\left(x_{2}\right)=\frac{2}{5}, \\
F_{1}\left(e_{2}\right)\left(x_{1}\right)=\frac{2}{7}, & F_{1}\left(e_{2}\right)\left(x_{2}\right)=\frac{3}{4},
\end{array}
$$

$$
\begin{array}{ll}
F_{2}\left(e_{1}\right)\left(x_{1}\right)=\frac{1}{3}, & F_{2}\left(e_{1}\right)\left(x_{2}\right)=\frac{1}{5}, \\
F_{2}\left(e_{2}\right)\left(x_{1}\right)=\frac{4}{5}, & F_{2}\left(e_{2}\right)\left(x_{2}\right)=\frac{1}{2}, \\
F_{3}\left(e_{1}\right)\left(x_{1}\right)=\frac{1}{3}, & F_{3}\left(e_{1}\right)\left(x_{2}\right)=\frac{1}{5}, \\
F_{3}\left(e_{2}\right)\left(x_{1}\right)=\frac{2}{7}, & F_{3}\left(e_{2}\right)\left(x_{2}\right)=\frac{1}{2}, \\
F_{4}\left(e_{1}\right)\left(x_{1}\right)=\frac{1}{3}, & F_{4}\left(e_{1}\right)\left(x_{2}\right)=\frac{2}{5}, \\
F_{4}\left(e_{2}\right)\left(x_{1}\right)=\frac{4}{5}, & F_{4}\left(e_{2}\right)\left(x_{2}\right)=\frac{3}{4}, \\
G_{1}\left(e_{1}\right)\left(y_{1}\right)=\frac{2}{5}, & G_{1}\left(e_{1}\right)\left(y_{2}\right)=0, \\
G_{1}\left(e_{2}\right)\left(y_{1}\right)=\frac{3}{4}, & G_{1}\left(e_{2}\right)\left(y_{2}\right)=0, \\
G_{6}\left(e_{2}\right)\left(y_{1}\right)=\frac{1}{2}, & G_{6}\left(e_{2}\right)\left(y_{2}\right)=0 . \\
G_{2}\left(e_{1}\right)\left(y_{1}\right)=\frac{1}{3}, & G_{2}\left(e_{1}\right)\left(y_{2}\right)=0, \\
G_{5}\left(e_{2}\right)\left(e_{1}\right)=\frac{1}{5}, & G_{2}\left(e_{2}\right)\left(y_{2}\right)=0, \\
G_{4}\left(e_{2}\right)\left(y_{1}\right)=\frac{1}{5}, & G_{6}\left(e_{1}\right)\left(y_{2}\right)=0, \\
G_{3}\left(e_{1}\right)\left(y_{1}\right)=\frac{1}{3}, & G_{4}\left(e_{2}\right)\left(y_{2}\right)=0, \\
G_{3}\left(e_{2}\right)\left(y_{1}\right)=\frac{1}{2}, & G_{3}\left(e_{1}\right)\left(y_{2}\right)=0, \\
\left.G_{3}\right)\left(y_{2}\right)=0, \\
G_{3}\left(e_{2}\right)\left(y_{2}\right)=0,
\end{array}
$$

Here $f^{-1}\left(G_{5}, E\right) \notin \tau$ and $f\left(\left(F_{3}, E\right)^{\prime}\right)$ is not a fuzzy soft closed set. Thus the mapping is not fuzzy soft continuous. Also it is not fuzzy soft closed mapping. But it is fuzzy soft open mapping.

Example 40. Let $E=\left\{e_{1}, e_{2}\right\}, X=\left\{x_{1}, x_{2}\right\}$ and $Y=\left\{y_{1}, y_{2}\right\}$. We define the mapping $f: X \rightarrow Y$ as $f\left(x_{1}\right)=f\left(x_{2}\right)=y_{2}$. Here the fuzzy soft sets are defined as follows:

$$
\begin{array}{ll}
F_{1}\left(e_{1}\right)\left(x_{1}\right)=\frac{2}{5}, & F_{1}\left(e_{1}\right)\left(x_{2}\right)=\frac{3}{5}, \\
F_{1}\left(e_{2}\right)\left(x_{1}\right)=1, & F_{1}\left(e_{2}\right)\left(x_{2}\right)=\frac{1}{2}, \\
F_{2}\left(e_{1}\right)\left(x_{1}\right)=\frac{1}{5}, & F_{2}\left(e_{1}\right)\left(x_{2}\right)=\frac{2}{5},
\end{array}
$$




$$
\begin{aligned}
& F_{2}\left(e_{2}\right)\left(x_{1}\right)=\frac{1}{3}, \quad F_{2}\left(e_{2}\right)\left(x_{2}\right)=\frac{1}{4}, \\
& F_{3}\left(e_{1}\right)\left(x_{1}\right)=\frac{3}{5}, \quad F_{3}\left(e_{1}\right)\left(x_{2}\right)=\frac{2}{5}, \\
& F_{3}\left(e_{2}\right)\left(x_{1}\right)=0, \quad F_{3}\left(e_{2}\right)\left(x_{2}\right)=\frac{1}{2}, \\
& F_{4}\left(e_{1}\right)\left(x_{1}\right)=\frac{1}{5}, \quad F_{4}\left(e_{1}\right)\left(x_{2}\right)=\frac{2}{5}, \\
& F_{4}\left(e_{2}\right)\left(x_{1}\right)=0, \quad F_{4}\left(e_{2}\right)\left(x_{2}\right)=\frac{1}{4}, \\
& F_{5}\left(e_{1}\right)\left(x_{1}\right)=\frac{3}{5}, \quad F_{5}\left(e_{1}\right)\left(x_{2}\right)=\frac{3}{5}, \\
& F_{5}\left(e_{2}\right)\left(x_{1}\right)=1, \quad F_{5}\left(e_{2}\right)\left(x_{2}\right)=\frac{1}{2}, \\
& F_{6}\left(e_{1}\right)\left(x_{1}\right)=\frac{3}{5}, \quad F_{6}\left(e_{1}\right)\left(x_{2}\right)=\frac{2}{5}, \\
& F_{6}\left(e_{2}\right)\left(x_{1}\right)=\frac{1}{3}, \quad F_{6}\left(e_{2}\right)\left(x_{2}\right)=\frac{1}{2}, \\
& F_{7}\left(e_{1}\right)\left(x_{1}\right)=\frac{2}{5}, \quad F_{7}\left(e_{1}\right)\left(x_{2}\right)=\frac{2}{5}, \\
& F_{7}\left(e_{2}\right)\left(x_{1}\right)=0, \quad F_{7}\left(e_{2}\right)\left(x_{2}\right)=\frac{1}{2}, \\
& F_{8}\left(e_{1}\right)\left(x_{1}\right)=\frac{2}{5}, \quad F_{8}\left(e_{1}\right)\left(x_{2}\right)=\frac{2}{5}, \\
& F_{8}\left(e_{2}\right)\left(x_{1}\right)=\frac{1}{3}, \quad F_{8}\left(e_{2}\right)\left(x_{2}\right)=\frac{1}{2}, \\
& G_{1}\left(e_{1}\right)\left(y_{1}\right)=1, \quad G_{1}\left(e_{1}\right)\left(y_{2}\right)=\frac{2}{5}, \\
& G_{1}\left(e_{2}\right)\left(y_{1}\right)=1, \quad G_{1}\left(e_{2}\right)\left(y_{2}\right)=\frac{1}{4}, \\
& G_{2}\left(e_{1}\right)\left(y_{1}\right)=1, \quad G_{2}\left(e_{1}\right)\left(y_{2}\right)=\frac{1}{5}, \\
& G_{2}\left(e_{2}\right)\left(y_{1}\right)=1, \quad G_{2}\left(e_{2}\right)\left(y_{2}\right)=\frac{1}{4}, \\
& G_{3}\left(e_{1}\right)\left(y_{1}\right)=1, \quad G_{3}\left(e_{1}\right)\left(y_{2}\right)=\frac{2}{5}, \\
& G_{3}\left(e_{2}\right)\left(y_{1}\right)=1, \quad G_{3}\left(e_{2}\right)\left(y_{2}\right)=0, \\
& G_{4}\left(e_{1}\right)\left(y_{1}\right)=1, \quad G_{4}\left(e_{1}\right)\left(y_{2}\right)=\frac{1}{5}, \\
& G_{4}\left(e_{2}\right)\left(y_{1}\right)=1, \quad G_{4}\left(e_{2}\right)\left(y_{2}\right)=0, \\
& G_{5}\left(e_{1}\right)\left(y_{1}\right)=1, \quad G_{5}\left(e_{1}\right)\left(y_{2}\right)=\frac{3}{5}, \\
& G_{5}\left(e_{2}\right)\left(y_{1}\right)=1, \quad G_{5}\left(e_{2}\right)\left(y_{2}\right)=\frac{1}{2},
\end{aligned}
$$

The mapping is a fuzzy soft closed mapping, but it is not both fuzzy soft open mapping and fuzzy soft continuous mapping.

Definition 41. Let $(X, \tau, E)$ and $\left(Y, \tau^{\prime}, E\right)$ be two fuzzy soft topological spaces and $f: X \rightarrow Y$ be a mapping. If $f$ is a bijection, fuzzy soft continuous and $f^{-1}$ is fuzzy soft continuous mapping, then $f$ is said to be fuzzy soft homeomorphism from $X$ to $Y$. When some homeomorphism $f$ exists, we say that $X$ is fuzzy soft homeomorphic to $Y$.

Theorem 42. Let $(X, \tau, E)$ and $\left(Y, \tau^{\prime}, E\right)$ be two fuzzy soft topological spaces and $f: X \rightarrow Y$ be a bijective mapping. Then the following conditions are equivalent:

(1) $f$ is a fuzzy soft homeomorphism,

(2) $f$ is a fuzzy soft continuous and fuzzy soft closed mapping,

(3) $f$ is a fuzzy soft continuous and fuzzy soft open mapping.

Proof. It is easily obtained.

\section{Conclusion}

The purpose of this paper is to discuss some important properties of fuzzy soft topological spaces. We investigate the properties of fuzzy soft interior and fuzzy soft closure of fuzzy sets. Besides, fuzzy soft continuous mapping, fuzzy soft open and fuzzy soft closed mappings, and fuzzy soft homeomorphism for fuzzy soft topological spaces are investigated, and some interesting results that may be of value for further research are obtained. We hope that this study will be useful for research in theoretical as well as in applicable directions.

\section{Acknowledgment}

Thanks are due to the referee for his/her useful comments.

\section{References}

[1] L. A. Zadeh, "Fuzzy sets," Information and Control, vol. 8, no. 3, pp. 338-353, 1965.

[2] K. T. Atanassov, "Intuitionistic fuzzy sets," Fuzzy Sets and Systems, vol. 20, no. 1, pp. 87-96, 1986.

[3] K. T. Atanassov, "Operators over interval valued intuitionistic fuzzy sets," Fuzzy Sets and Systems, vol. 64, no. 2, pp. 159-174, 1994.

[4] M. B. Gorzałczany, "A method of inference in approximate reasoning based on interval-valued fuzzy sets," Fuzzy Sets and Systems, vol. 21, no. 1, pp. 1-17, 1987.

[5] Z. Pawlak, "Rough sets," International Journal of Computer \& Information Sciences, vol. 11, no. 5, pp. 341-356, 1982. 
[6] D. Molodtsov, "Soft set theory-irst results," Computers and Mathematics with Applications, vol. 37, no. 4-5, pp. 19-31, 1999.

[7] P. K. Maji, R. Biswas, and A. R. Roy, "Soft set theory," Computers and Mathematics with Applications, vol. 45, no. 4-5, pp. 555-562, 2003.

[8] P. K. Maji, A. R. Roy, and R. Biswas, "An application of soft sets in a decision making problem," Computers and Mathematics with Applications, vol. 44, no. 8-9, pp. 1077-1083, 2002.

[9] D. Chen, E. C. C. Tsang, D. S. Yeung, and X. Wang, "The parameterization reduction of soft sets and its applications," Computers and Mathematics with Applications, vol. 49, no. 5-6, pp. 757-763, 2005.

[10] F. Feng, Y. B. Jun, and X. Zhao, "Soft semirings," Computers and Mathematics with Applications, vol. 56, no. 10, pp. 2621-2628, 2008.

[11] H. Aktaş and N. Çağman, "Soft sets and soft groups," Information Sciences, vol. 177, no. 13, pp. 2726-2735, 2007.

[12] Q. M. Sun, Z. L. Zhang, and J. Liu, "Soft sets and soft modules," Lecture Notes in Computer Science, vol. 5009, pp. 403-409, 2008.

[13] M. I. Ali, F. Feng, X. Liu, W. K. Min, and M. Shabir, "On some new operations in soft set theory," Computers and Mathematics with Applications, vol. 57, no. 9, pp. 1547-1553, 2009.

[14] B. Ahmad and A. Kharal, "On fuzzy soft sets," Advances in Fuzzy Systems, vol. 2009, Article ID 586507, 6 pages, 2009.

[15] P. K. Maji, R. Biswas, and A. R. Roy, “Fuzzy soft sets," Journal of Fuzzy Mathematics, vol. 9, no. 3, pp. 589-602, 2001.

[16] A. Kharal and B. Ahmad, "Mappings on fuzzy soft classes," Advances in Fuzzy Systems, vol. 2009, Article ID 407890, 6 pages, 2009.

[17] X. Yang, X. Yang, T. S. Lin, J. Yang, Y. Li, and D. Yu, "Combination of interval-valued fuzzy set and soft set," Computers and Mathematics with Applications, vol. 58, no. 3, pp. 521-527, 2011.

[18] M. Shabir and M. Naz, "On soft topological spaces," Computers and Mathematics with Applications, vol. 61, no. 7, pp. 1786-1799, 2011.

[19] B. Tanay and M. B. Kandemir, "Topological structure of fuzzy soft sets," Computers and Mathematics with Applications, vol. 61, no. 10, pp. 2952-2957, 2011.

[20] C. L. Chang, "Fuzzy topological spaces," Journal of Mathematical Analysis and Applications, vol. 24, no. 1, pp. 182-190, 1968.

[21] B. Pazar Varol and H. Aygun, "Fuzzy soft topology", Hacettepe Journal of Mathematics and Statistics, vol. 41, no. 3, pp. 407-419, 2012. 


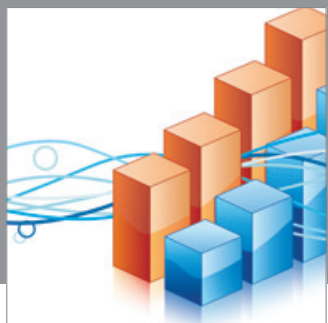

Advances in

Operations Research

mansans

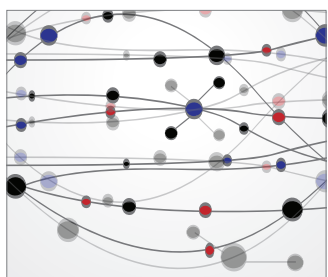

The Scientific World Journal
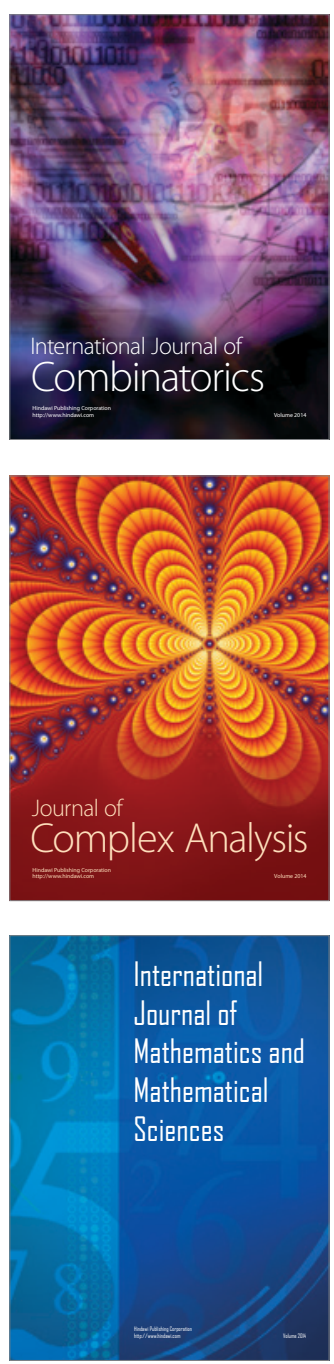
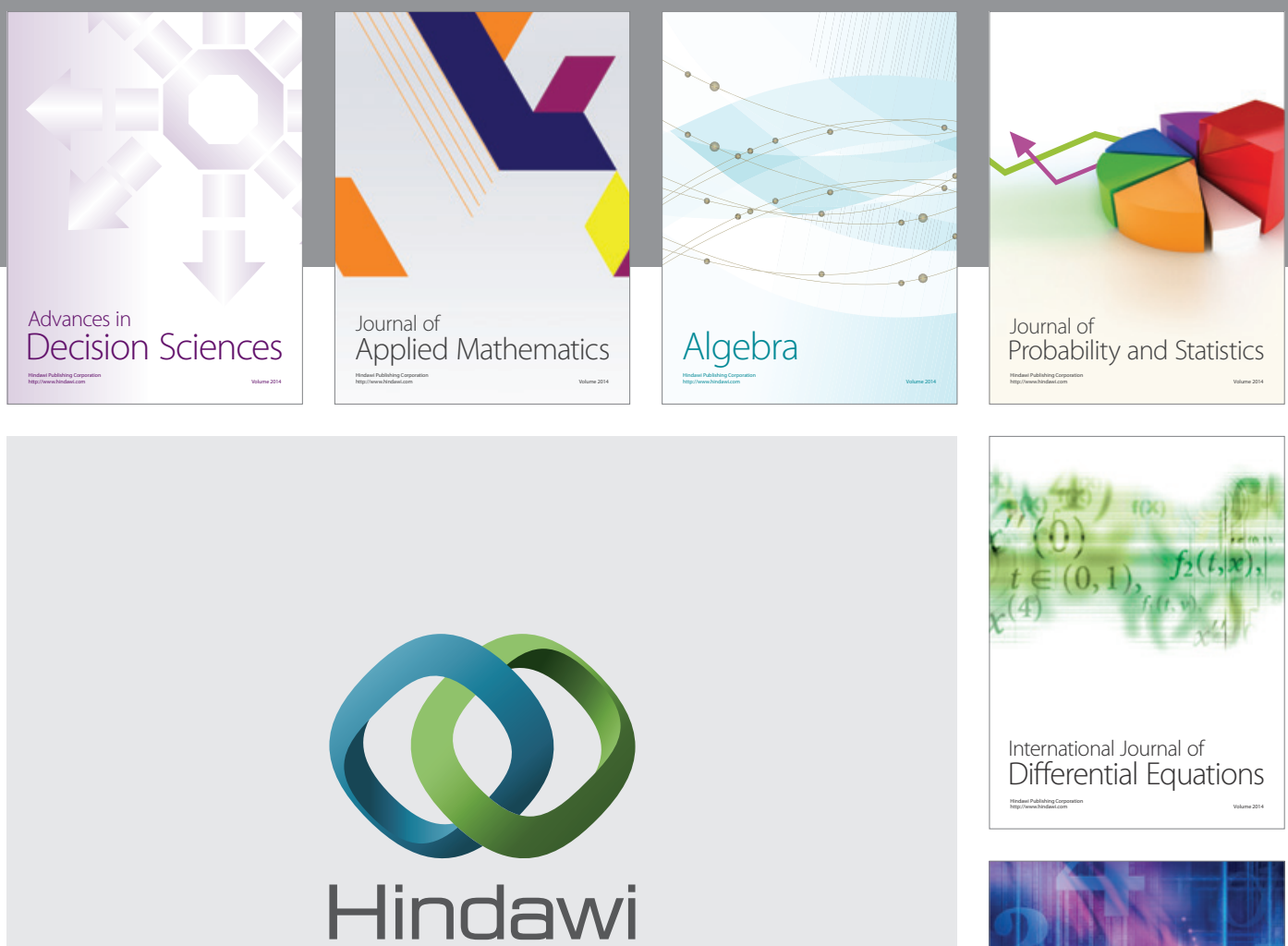

Submit your manuscripts at http://www.hindawi.com
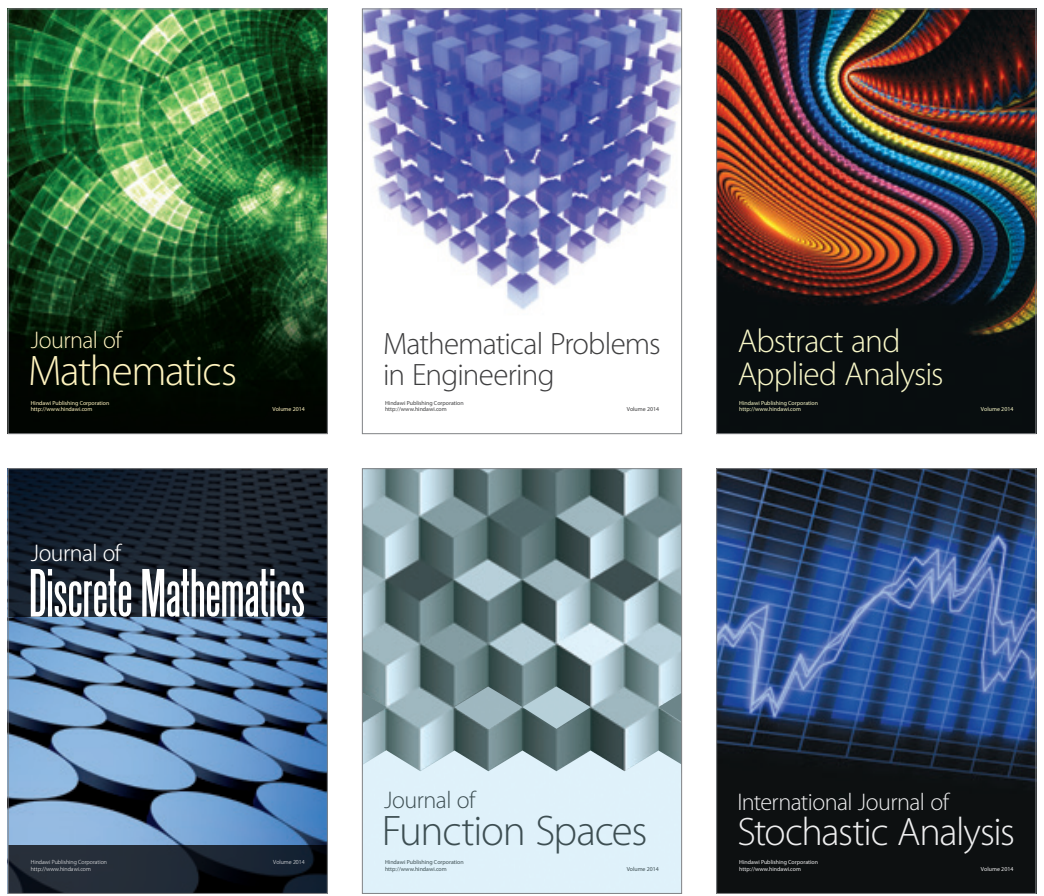

Journal of

Function Spaces

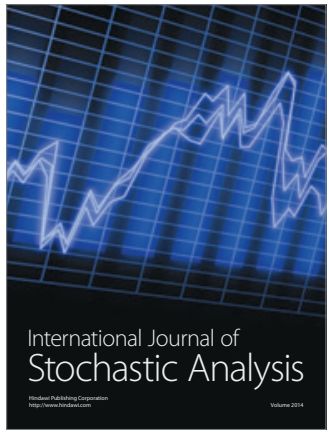

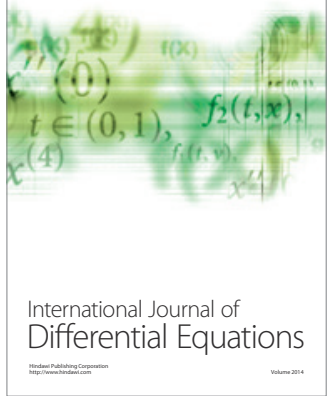
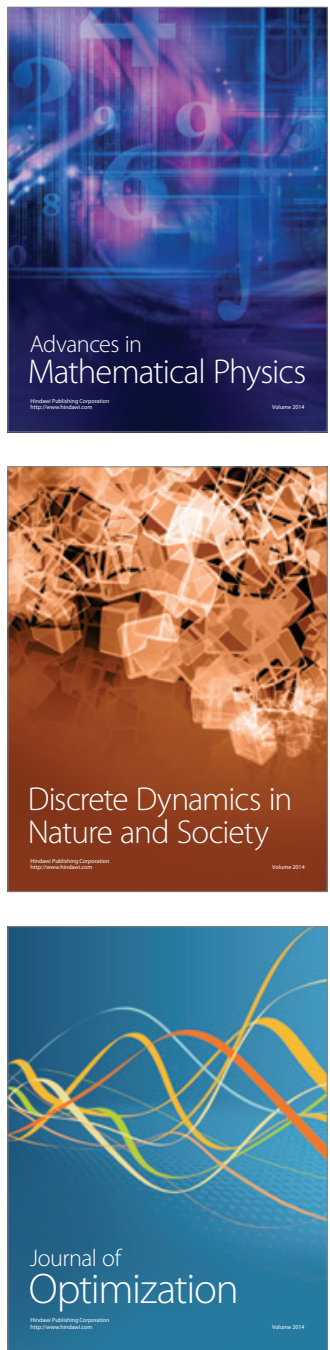